\title{
Nanoscale
}

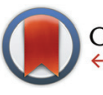

CrossMark \& click for updates

Cite this: Nanoscale, 2016, 8, 15288

\section{Nanoengineering a library of metallic nanostructures using a single microfluidic reactor $\dagger$}

\author{
Víctor Sebastián ${ }^{\mathrm{a}, \mathrm{b}}$ and Klavs F. Jensen ${ }^{\star a}$
}

Microfluidic synthesis in a microfabricated reactor enables fast and facile synthesis of a wide library of metallic nanostructures: monometallic, bimetallic, anisotropic growth and heterostructures. Specific nanostructures are realized by selection of flow pattern and synthesis parameters. The technique is shown to have advantages over conventional batch technologies. Not only does it allow faster scalable synthesis, but also realization of nanostructures hitherto not reported such as $\mathrm{Pt}-\mathrm{Ru}, \mathrm{Pt}-\mathrm{Ni}$ and $\mathrm{Pt}-\mathrm{Co}$ nanodendrites, Pt-Pd heterostructures, $\mathrm{Ag}-\mathrm{Pd}$ core-shell NPs, $\mathrm{Au}-\mathrm{Pd}$ nanodumbbells and $\mathrm{Au}-\mathrm{Pd}$ nanosheets.

Received 20th May 2016,

Accepted 2nd August 2016

DOI: $10.1039 / c 6 n r 04104 c$

www.rsc.org/nanoscale

\section{Introduction}

The synthesis of nanomaterials for electronic, optical and medical applications requires tight control of nucleation and growth processes to realize materials with desired features and properties. ${ }^{1,2}$ Significant efforts have been devoted to improving size distributions and quality beyond those obtained with conventional batch reactors. ${ }^{3,4}$ Highly monodisperse nanoparticles (NPs) are commonly produced by two stage synthesis: polydisperse nanoparticles are first synthesized using standard synthetic routes and afterwards, NPs of a given particle size are isolated by size classification methods and repeated recrystallizations. ${ }^{5}$ These approaches may be appropriate for small-scale production of nanostructures, but they are typically too laborious and have too low yields for large-scale production. ${ }^{3}$

Continuous flow technologies offer potential advantages over batch synthesis, including feedback control of temperature and feed streams, reproducibility, potential for sensor integration for in situ synthesis monitoring, rapid screening of parameters and easier scale-up. ${ }^{6,7}$ The larger surface to volume ratio of microfluidic reactors (MR) enhance mass and heat transfer, ${ }^{8}$ but present challenges in terms of wall deposition and particle agglomeration and blockage of the flow path. ${ }^{9}$ The ability to work at elevated temperatures and pressures

\footnotetext{
${ }^{a}$ Department of Chemical Engineering, Massachusetts Institute of Technology, 77 Massachusetts Avenue, Cambridge, MA 02139, USA. E-mail: kfjensen@mit.edu ${ }^{b}$ Department of Chemical Engineering, Aragon Institute of Nanoscience (INA), University of Zaragoza, Campus Rio Ebro, 50018 Zaragoza, Spain and Networking Research Center on Bioengineering, Biomaterials and Nanomedicine (CIBER-BBN), 28029 Madrid, Spain

$\dagger$ Electronic supplementary information (ESI) available: Supporting figures and experimental information. See DOI: 10.1039/c6nr04104c
}

present opportunities for using environmentally benign solvent and accelerating nanoparticle synthesis. ${ }^{6}$

Herein we report a versatile synthesis platform based in a single microfluidic reactor to obtain a broad library of metal nanostructures in a continuous fashion. Although micro and meso scale flow reactors have been used to improve particle size distribution of nanomaterials, ${ }^{10,11}$ to promote the formation of nanocoatings ${ }^{12,13}$ and to effect shape control, ${ }^{14-21}$ this report is the first time, to our knowledge, that same microfluidic system is applied to control shape, size and chemical composition of nanomaterials. The library of nanostructures obtained comprises monodisperse monometallic and bimetallic NPs, Pt-based nanodendrites, nano core-shell structures, nanocubes, Janus nanostructures, dumbbells, nanorods and nanosheets. The microfluidic continuous synthesis at elevated temperature and pressures produces nanostructures on the time scales of seconds to minutes in contrast to the hours reported for traditional batch. These results are realized by modification of precursor chemistry and synthesis conditions (temperature, pressure, reactants, residence time and flow conditions). Moreover, new nanostructures are synthesized under conditions difficult to achieve in batch.

\section{Results and discussion}

Microfabrication techniques produced the silicon-Pyrex MR (Fig. 1) used in the continuous synthesis of the library of NPs. $^{22}$ The unit could withstand high pressures $(6 \mathrm{MPa})$ and high temperature $\left(350^{\circ} \mathrm{C}\right)$, and allowed easy optical access from Pyrex side. In order to control the nucleation and growth steps, the device had two zones, a cold inlet/outlet mixing area and a hot reaction area. ${ }^{23,24}$ Mixing of precursors in the cold area before entering to the reaction zone enabled us to achieve 


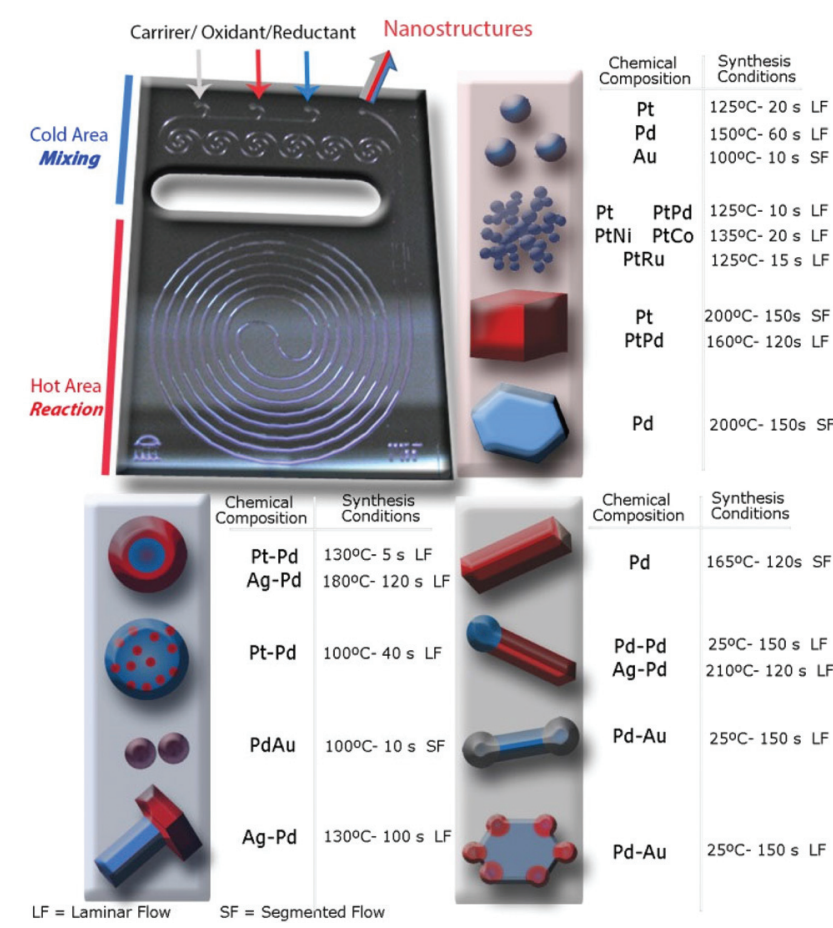

Fig. 1 Silicon-Pyrex microfluidic reactor device used to synthesize the library of nanostructures. Schematic of the library of metallic nanostructures obtained and their synthesis conditions: monometallic and bimetallic spherical nanoparticles, nanodendrites, nanocubes, core-shell nanoparticles, Janus nanostructures, dumbbells, nanorods and nanosheets.

monodisperse nanostructures. The outlet stream also passed through the cold area to quench further nanomaterials growth. The reaction zone $(100 \mu \mathrm{L})$ was a long spiral channel (400 $\mu \mathrm{m}$ width) designed to prevent solid accumulation and clogging. Segmented flow was generated by the separation of small liquid droplets at the nanoliter scale with gas slugs to enhance the proper reagents mixing (Fig. S1 in ESI $\dagger$ ). ${ }^{23}$ The flow pattern in the reactor could be switched from laminar to segmented flow through the inlet labelled as carrier, in order to overcome dispersion problems associated with laminar flow at short residence times. ${ }^{25}$

\section{Synthesis of metallic nanoparticles}

Access to active sites is a key issue in production of NP for catalysis. Strong capping agents promote monodispersed nanoparticle synthesis, but also can compete with reactants and products for adsorption sites, altering the catalytic action of the particle. ${ }^{26}$ In that case, the use of a weak capping agent is preferred, but synthesis of small monodisperse NPs becomes challenging because uncontrolled mixing promotes particle agglomeration and heterogeneous size distribution. We were able to synthesize different monodisperse noble metal NPs $(<5 \mathrm{~nm})$ by using the MR and a weak capping agent tetradecyltrimethylammonium bromide $-\mathrm{TTAB}^{26}$ (see ESI $\dagger$ for experimental details).
Reduction of $\mathrm{Pt}^{2+}$ ions with a strong agent such as sodium borohydride promoted a fast nucleation process that produced individual small NPs at reaction temperatures over $100{ }^{\circ} \mathrm{C}$ and reaction times shorter than $60 \mathrm{~s}$ (Fig. S2 and S3 $\dagger$ ). Monodisperse Pt NPs, $3.3 \mathrm{~nm} \pm 0.2$, were obtained at $125^{\circ} \mathrm{C}$ and $20 \mathrm{~s}$ residence time, $t_{\mathrm{R}}$, (Fig. $\mathrm{S} 2 \mathrm{a}$ and $\mathrm{b} \dagger$ ). The nucleation and growth stages were sensitive to synthesis temperature and residence time, with the size distribution of Pt NPs broadening as the temperature and residence time increased in the range of $125{ }^{\circ} \mathrm{C}-200{ }^{\circ} \mathrm{C}$ and $5 \mathrm{~s}-60 \mathrm{~s}$, respectively (Fig. S3a and b $\dagger$ ). By the controlled addition of ethanol in the reaction mixture, the size of the Pt NPs could be tuned even to small dimensions $(2.0 \mathrm{~nm} \pm 0.2)$ (Fig. 2a, S2b and S3c $\dagger$ ). Ethanol is a mild reducing agent that promote monodisperse distribution of small nanoparticles by both modifying the saturation concentration during the nucleation process and increasing the reduction rate of sodium borohydride. ${ }^{27}$

Using the polyol method with ethylene glycol as reducing agent at $150{ }^{\circ} \mathrm{C}$ and $60 \mathrm{~s}$ residence time produced monodisperse Pd NPs under $3 \mathrm{~nm}$ (Fig. $2 \mathrm{~b}$ and S2d $\dagger$ ). Sodium borohydride as reducing agent instead of ethylene glycol, yielded palladium agglomerates over the entire range of reaction conditions tested (Fig. S2c广). These findings demonstrate the importance of using the proper solvent and reducing agent to realize continuous MR synthesis of monodisperse NPs without agglomeration and clogging.

Growth of Au NPs is normally achieved by an initial and rapid stage of gold precursor reduction into gold nuclei within less than $200 \mathrm{~ms}$, after which the nuclei grow due to a
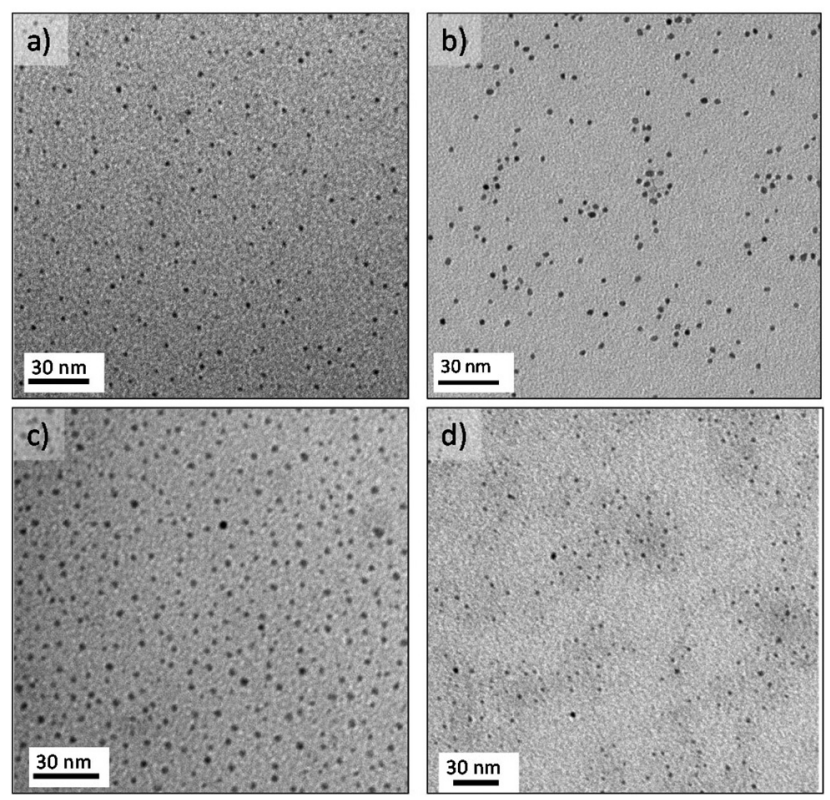

Fig. 2 TEM images of metallic spherical NPs produced under different reaction conditions. (a) Pt NPs collected in laminar flow at $125^{\circ} \mathrm{C}$ and $t_{\mathrm{R}}$ $=20 \mathrm{~s}$. (b) Pd NPs collected in laminar flow at $150{ }^{\circ} \mathrm{C}$ and $t_{\mathrm{R}}=60 \mathrm{~s}$. (c) Au NPs. (d) AuPd alloy NPs, both collected in air segmented flow at $100{ }^{\circ} \mathrm{C}$ and $t_{\mathrm{R}}=10 \mathrm{~s}$. 
coalescence process. ${ }^{28}$ Thus, the mixing time must be shorter than $200 \mathrm{~ms}$ to obtain monodisperse Au NPs. However, representative TEM images show broad size distributions of $\mathrm{Au}$ NPs produced across the entire range of synthesis conditions (Fig. S2e†). Thus, the fast reactions in a MR are not sufficient to realize narrow size distributions. The relative values of the kinetic rate and the rate of mixing, together with axial dispersion in single phase flow, decide the extent of polydispersity in the nanocrystals formation. ${ }^{23}$ Flow segmentation increases the cross stream mixing, reducing dispersion, to generate uniform sized Au NPs. ${ }^{29,30}$ Fig. $2 \mathrm{c}$ shows a monodisperse distribution of gold nanoparticles, $2.8 \pm 0.2 \mathrm{~nm}$, obtained at $100{ }^{\circ} \mathrm{C}$ with an air segmented flow and a residence time of $10 \mathrm{s.}^{23}$ The particle size of collected Au NPs at a residence time larger than $10 \mathrm{~s}$ were not controlled because the low slip velocity between slugs lead to poor mixing inside the slugs, which as a consequence leads to a wider particle size distribution (Fig. S3d $\dagger)^{23}$

Gold-palladium bimetallic NPs have received significant attention for their special catalytic properties, but it is challenging not end up with segregated populations of monometallic NPs when synthesizing small monodisperse NPs $(<5 \mathrm{~nm}) \cdot{ }^{31}$ By using the previous mentioned gas segmented flow, monodisperse AuPd bimetallic NPs $(3.7 \pm 0.3 \mathrm{~nm})$ resulted at the same conditions for the pure Au counterpart. The UV-visible spectra of the bimetallic NPs did not show the surface plasmon signature of pure $\mathrm{Au}$ nanoparticles in the 500-520 nm range, suggesting the synthesis of bimetallic nanoparticles (Fig. S2f $\dagger$ ). This phenomenon is consistent with previous observations ${ }^{19,32,33}$ of a group 10 metal $\left(\mathrm{d}^{8} \mathrm{~s}^{2}\right)$ in the bimetallic alloyed NPs suppressing the surface plasmon resonance energies of group 11 metals $\left(\mathrm{d}^{10} \mathrm{~s}^{1}\right)$ and implies the formation of $\mathrm{Au} / \mathrm{Pt}$ alloyed NPs.

\section{Synthesis of platinum and palladium heterostructures}

Platinum and palladium are important catalysts for many industrial processes. ${ }^{34}$ When $\mathrm{Pd}$ is added to $\mathrm{Pt}$, a change in the electronic structure can lead to activity enhancement for particular reactions. ${ }^{35}$ Both metals adopt the highly symmetrical face-centered-cubic (fcc) crystal structure, and the two metals have a very close lattice match promoting alloying (Pd and Pt have a lattice mismatch of only $0.77 \%$ ). Thus, it is challenging to obtain nanostructures with Pt and Pd as segregated phases in a single step. In this work, we realized for the first time different Pt-Pd nanostructures in a continuous and single step process and using the advantages of microfluidics and some shape control strategies ${ }^{36}$ (Fig. 3). Growth at $130{ }^{\circ} \mathrm{C}$ with residence time as short as $5 \mathrm{~s}$ produced Pt-Pd core-shell nanostructures, where Pt is located in the core (Fig. 3a). Modifying the Pt/Pd precursor flow ratios tuned the shell thickness and particles size distribution, obtaining a thick shell at a large $\mathrm{Pt} / \mathrm{Pd}$ ratio (Fig. $\mathrm{S} 4 \dagger$ ). At these conditions, Pt with a reduction potential higher that Pd nucleated faster and Pd grew heterogeneously on the Pt cores. The high-speed flows in the microchannels yielded fast multi-fluid lamination, which appeared to be a requirement for obtaining the core-shell
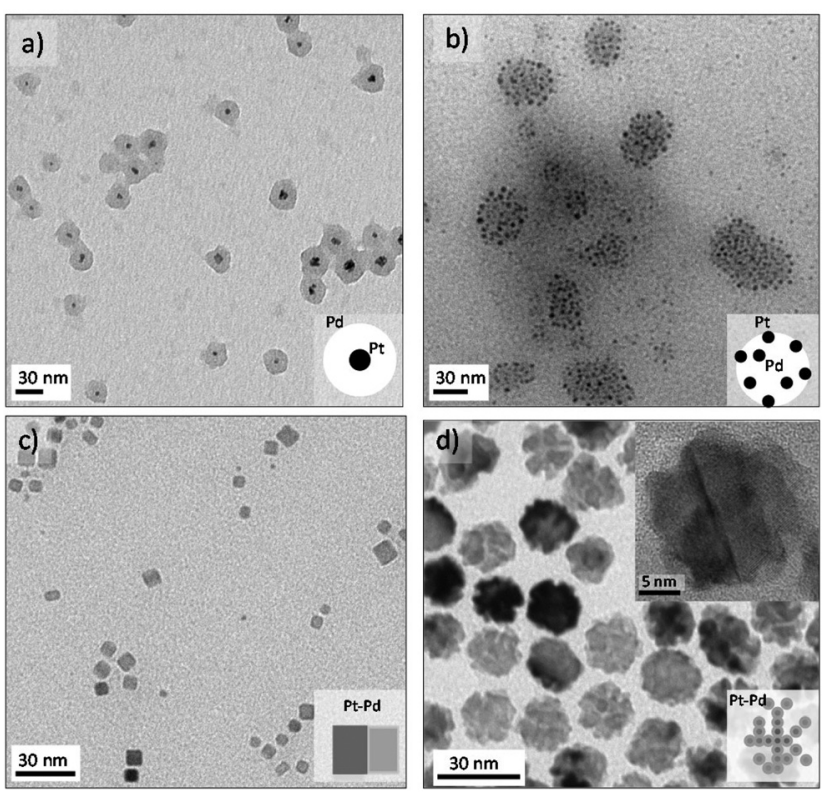

Fig. 3 TEM images of metallic platinum-palladium nanostructures produced under different reaction conditions. (a), PtPd core-shell nanostructure, platinum located at the core, collected at $130{ }^{\circ} \mathrm{C}$ and $t_{\mathrm{R}}=$ $5 \mathrm{~s}$. (b), Pd NPs decorated with Pt NPs collected at $100{ }^{\circ} \mathrm{C}$ and $t_{\mathrm{R}}=$ $40 \mathrm{~s}$. (c) PtPd alloy nanocubes collected at $160{ }^{\circ} \mathrm{C}$ and $t_{\mathrm{R}}=120 \mathrm{~s}$. (d) PtPd nanodendrites, collected at $125^{\circ} \mathrm{C}$ and $t_{\mathrm{R}}=20 \mathrm{~s}$.

structure. Pt and Pd agglomerates were obtained at residence times larger than $20 \mathrm{~s}$ (Fig. S4a†). The addition of ethanol to the crystallization mixture slowed down the platinum nucleation, switching the crystallization pattern between Pt and Pd. Under those conditions, Pd crystallized rapidly and Pd spherical particles decorated with small $2.1 \mathrm{~nm}$ Pt NPs resulted (Fig. 4b). The molar composition of both metallic precursors controlled the density of the Pt NPs on the Pd cores (Fig. S5a and $b \dagger$ ).

Pt-Pd alloyed nanocubes were obtained using the before mentioned polyol method under an air segmented flow to promote the oxidative etching (Fig. $3 \mathrm{c}$ and $\mathrm{S} 5 \mathrm{c} \uparrow)^{19}{ }^{19}$ The oxidative etching enables the selective etching of twinned particles, promoting the formation of cubic shape NPs. ${ }^{37}$ Further details about oxygen slug flow and oxidative etching appear below in the anisotropic growth section.

Bimetallic single crystal PtPd nanodendrites $(17 \pm 2 \mathrm{~nm})$ were obtained by regulating the reduction kinetics with a mild reducing agent, ascorbic acid, which promotes dendritic growth (Fig. 3d and S5d广). This synthesis approach will be discussed in the section on synthesis of 3D dendritic nanostructures.

\section{Synthesis of palladium and silver heterostructures}

Palladium and silver nanostructures were also nanoengineered with good morphology control by using the same MR. Ag-Pd Janus-like NPs were obtained in continuous flow at $130{ }^{\circ} \mathrm{C}$ and $100 \mathrm{~s}$ residence time, using the polyol synthesis method (Fig. 4a). Segregation of individual palladium and silver 

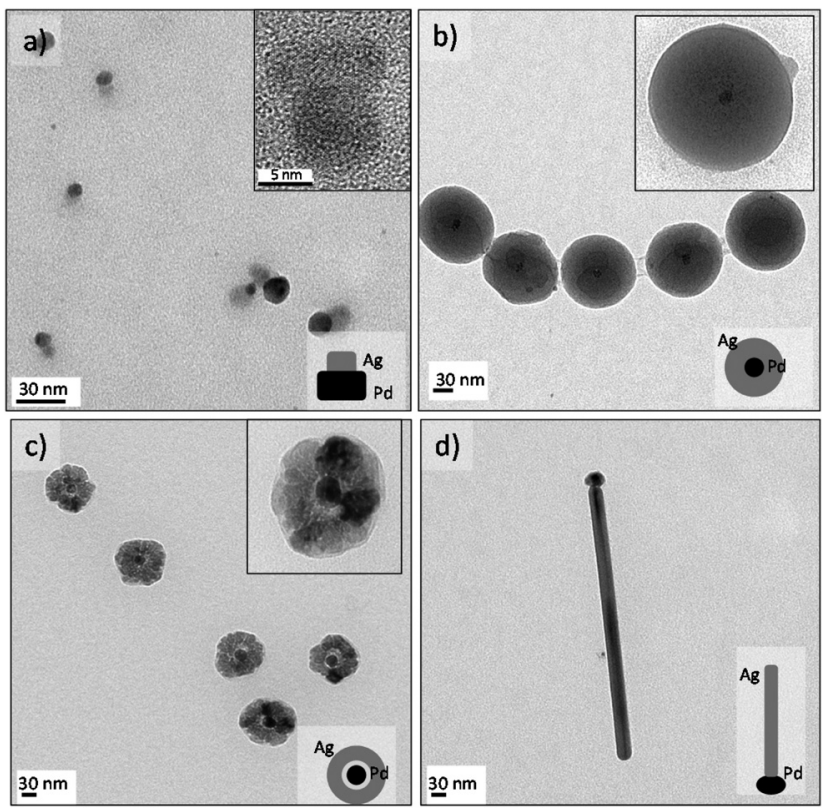

d)

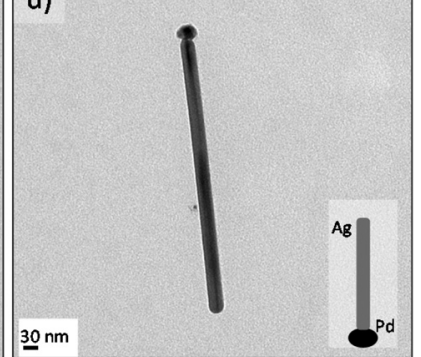

Fig. 4 TEM images of metallic silver-palladium nanostructures produced under different reaction conditions. (a) AgPd semi-Janus nanostructure, collected at $130{ }^{\circ} \mathrm{C}$ and $t_{\mathrm{R}}=100 \mathrm{~s}$. (b) and (c) AgPd core-shell nanostructures collected at $t_{\mathrm{R}}=120 \mathrm{~s}$, and $160^{\circ} \mathrm{C}$ and $180^{\circ} \mathrm{C}$, respectively. (d) Ag nanowire with a Pd head, nanostructure collected at $210^{\circ} \mathrm{C}$ and $t_{\mathrm{R}}=120 \mathrm{~s}$.

particles resulted when the synthesis temperature was lower than $130{ }^{\circ} \mathrm{C}$ or with use of another reducing agent, such as sodium borohydride or ascorbic acid (Fig. S6a and $\mathrm{b} \dagger$ ). The HRTEM micrograph shows that Pd and Ag Janus-like nanostructure form via epitaxial growth and with high crystallinity (Fig. S5c and $\mathrm{d} \dagger$ ).

Core-shell morphology resulted by increasing the Ag to Pd molar ratio at high temperature, $160{ }^{\circ} \mathrm{C}$ (Fig. 4b). Under those conditions, Pd nucleated faster and $\mathrm{Ag}$ nucleated on it to form a shell ultimately. At $180{ }^{\circ} \mathrm{C}$, we observed both a hollow halo between the core and the shell and porosity in the $\mathrm{Ag}$ shell, suggesting a galvanic replacement reaction (Fig. 4c).

“Tadpole" like Ag-Pd NPs were observed to form at a temperature higher than $210{ }^{\circ} \mathrm{C}$, where silver grew as a nanowire and palladium was the seed activating the growth (Fig. 4d). These results are in agreement with the work of Xia et al. ${ }^{38}$ who have recently reported the production of $\mathrm{Ag}$ nanorods using Pd decahedral seeds. Silver atoms only deposited along one side of the Pd decahedral seed to generate $\mathrm{Ag}$ nanorods with the Pd seed being positioned at one of its two ends. ${ }^{38}$ For reaction temperatures exceeding $220{ }^{\circ} \mathrm{C}$, the microfluidic channels completely clogged, presumably because of the tadpole dimensions (Fig. 4d and S6e and $\mathrm{f} \dagger$ ).

\section{Synthesis of 3D dendritic nanostructures}

The synthesis of metal 3D dendritic nanostructures has received attention for catalytic applications because of these nanoarchitectures combine a high surface area with active
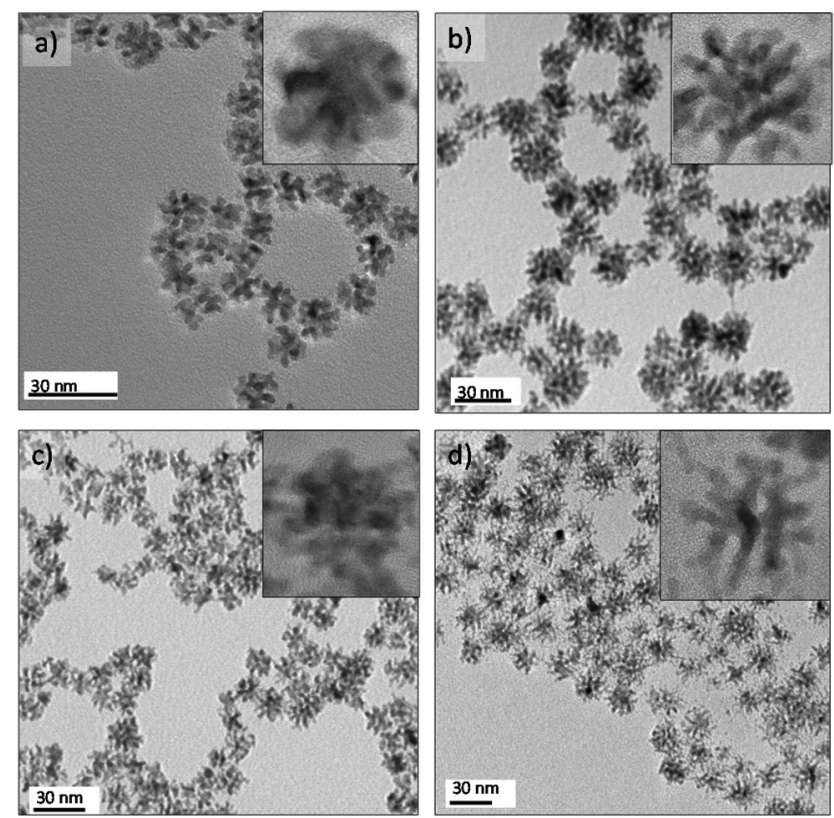

Fig. 5 TEM images of bimetallic platinum nanodendrites (NDs). (a) Pt NDs, produced at $125^{\circ} \mathrm{C}$ and $t_{R}=20 \mathrm{~s}$. (b) PtNi NDs, collected at $135^{\circ} \mathrm{C}$ and $t_{\mathrm{R}}=20 \mathrm{~s}$., (c) PtCo NDs obtained at $135^{\circ} \mathrm{C}$ and $t_{\mathrm{R}}=20 \mathrm{~s}$. (d) PtRu NDs collected at $125^{\circ} \mathrm{C}$ and $t_{\mathrm{R}}=15 \mathrm{~s}$.

edges. ${ }^{39-43}$ However, availability of scalable procedures to realize large-scale synthesis has limited their applications. Here we report, for the first time, the continuous flow synthesis of Pt and Pt-M bimetallic dendritic nanostructures of $\mathrm{Pd}, \mathrm{Ni}, \mathrm{Co}$ and Ru (Fig. 3d and 5). The metallic nanodendrites (NDs) were obtained via a one-step aqueous phase reaction with a mild reducing agent (ascorbic acid) and by controlling the nucleation and growth through three critical variables: reaction temperature, residence time and $\mathrm{pH}$. The excellent synthesis control afforded by microfluidics produced NDs with an acceptable size distribution in residence times as small as $20 \mathrm{~s}$. The slow nucleation rate of Pt in the presence of ascorbic acid could be an explanation for the differences from monodisperse Pt NPs obtained with $\mathrm{NaBH}_{4}$ as reducing agent (Fig. 2a). Lim et al. $^{44}$ stated that the growth mechanism of Pt NDs is based on oriented attachment of the initially Pt formed particles, suggesting that discrete Pt nanoparticles were fused to form branches.

The continuous flow synthesis occurred in seconds, much faster than the hour long time scales reported for batch synthesis. ${ }^{40,41}$ The NDs were characterized by HRTEM to gain insight into the crystallinity and nanobranches arrangement. HRTEM micrographs reveal that the ND morphologies depend on the metals incorporated. The branching is less favoured in $\mathrm{Pt}-\mathrm{Pd}$ NDs(Fig. S5d $\dagger$ ). In contrast, Pt-Ru and Pt-Ni NDs are highly branched (Fig. S7e and $\mathrm{f} \dagger$ ). Pt-Ni and Pt-Co NDs were magnetic so they can in principle be collected and recycled by applying a magnetic field. ${ }^{24}$ The bimetallic composition was confirmed by energy-dispersive X-ray spectroscopy analysis (Fig. S7g $\dagger$ ). The lattice fringes reveal the high crystallinity of 
the NDs and show a continuous lattice in Pt, Pt-Ni, Pt-Co and $\mathrm{Pt}-\mathrm{Ru}$ (Fig. $\mathrm{S} 7 \dagger$ ). Domain boundaries were clearly observed in Pt-Ni NDs, supporting the idea that two different mechanisms could occur: epitaxial growth by oriented attachment ${ }^{44}$ in Pt, $\mathrm{Pt}-\mathrm{Co}, \mathrm{Pt}-\mathrm{Ru}$ and $\mathrm{Pt}-\mathrm{Pd}$ and random aggregation of $\mathrm{Pt}$ and $\mathrm{Ni}$ nuclei in Pt-Ni NDs.

\section{Synthesis of anisotropic nanostructures}

The microfluidic reactor was also applied in kinetically controlled synthesis of nanostructures under short synthesis times. ${ }^{19}$ Anisotropic growth of palladium can be obtained in two different environments, air and carbon monoxide, obtaining nanostructures with a different morphology. ${ }^{45}$ The combination of oxygen from air and $\mathrm{Cl}^{-}$ions are a powerful etchant to selectively control the growth of single crystals seeds rather than twinned seeds. ${ }^{46}$ The oxidative etching preferentially affect twin seeds because of the presence of defect zones on the surface, being the zero-valent metal atoms oxidized back to ions. Nevertheless, not only the oxidative etching is required to anisotropically grow Pd nanostructures, but also a proper reduction rate and a selective capping agent to promote both of them the atomic addition on a specific facet. ${ }^{47}$

The gas-liquid segmented approach was proposed as a tool to control the oxidative etching ${ }^{19}$ because air slugs promoted the oxygen transfer from the gas to the liquid phase and it enables an easy phase-product separation after synthesis. Furthermore the reactant injection in separated streams allowed obtaining a fine control on the oxidative etching and reducing rate. The palladium atomic addition was also controlled by the synthesis temperature, achieving an atomic addition sufficiently fast to produce elongated nanostructures and preventing the formation of low aspect nanostructures or cubooctahedrons. ${ }^{19}$ Fig. 6a shows the Pd nanorods obtained at $165{ }^{\circ} \mathrm{C}$ and 2 minutes residence time, using $\mathrm{Br}-$ ions as capping agents to promote the formation of $\{100\}$ and $\{110\}$ facets. The nanorod aspect ratio could be varied to obtain approximately a value of $4.5-6 .^{19}$

Carbon monoxide-liquid segmented flow was also applied to modify the anisotropic growth of palladium into a sheet-like shape. ${ }^{45}$ Carbon monoxide has the double role of being a reducing agent and a selective capping agent. ${ }^{15}$ The chemisorption of CO monoxide on $\{111\}$ palladium facets can provide a means for controlling the atomic addition, hindering the growth along the [111] direction. ${ }^{48}$ Such hindered growth promotes the formation of a sheet-like structure. ${ }^{48,49}$ Carbon monoxide reduce $\mathrm{Pd}$ ions forming a $\mathrm{Pd}$ carbonyl complex, which collapses into clusters that are added onto given facets. ${ }^{49,50}$ Bromide ions were selected to define the sheet-like structure in a hexagonal shape. Fig. 6d shows a TEM micrograph of the palladium nanosheets obtained at $100{ }^{\circ} \mathrm{C}$ and $150 \mathrm{~s}$ residence time. The nanosheets presented sharp edges with a homogenous size distribution, edge lengths of $14 \pm$ $1.4 \mathrm{~nm}$. Fig. 6d shows a stack of nanosheets with ultrathin sheets of $1.2 \mathrm{~nm}$. The high aspect ratio between the edge length and thickness of the palladium nanosheets determine the optical properties of this nanostructure, ${ }^{48}$ producing a

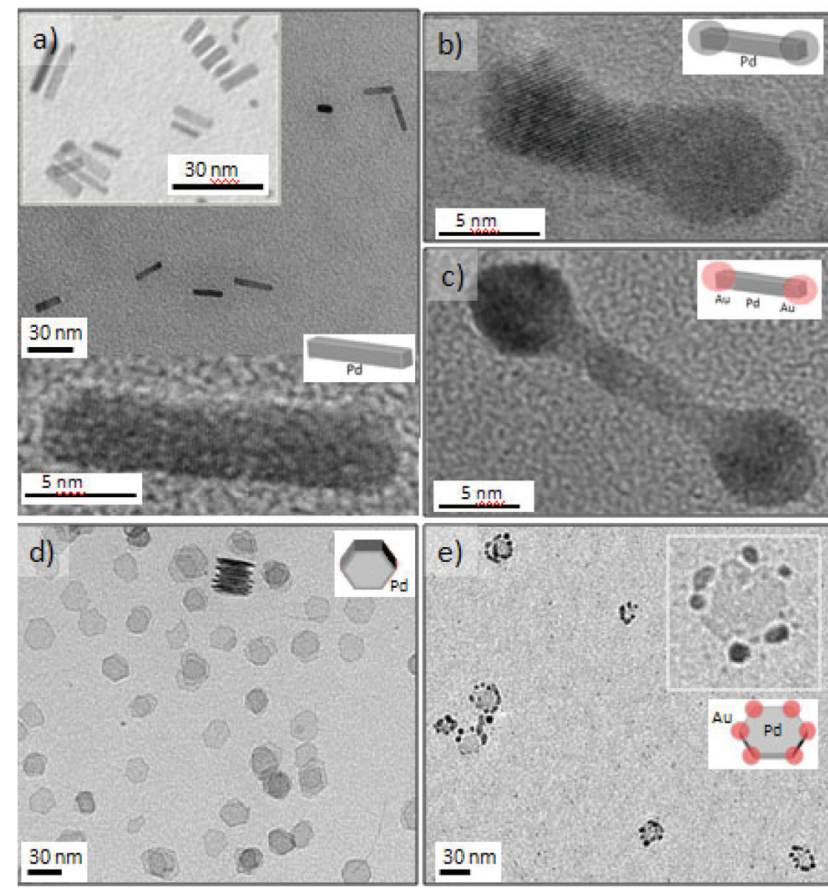

Fig. 6 TEM images of metallic Pd nanostructures produced under different reaction conditions in a segmented flow. (a) Pd nanorods collected in air segmented flow at $165^{\circ} \mathrm{C}$ and $t_{\mathrm{R}}=120 \mathrm{~s}$ (b) Pd nanodumbbells collected under ultrasound radiation at $25^{\circ} \mathrm{C}$ and $t_{R}=150 \mathrm{~s}$. (c) $\mathrm{PdAu}$ nanodumbbells, Au located at the sides, collected under ultrasound radiation at $25{ }^{\circ} \mathrm{C}$ and $t_{\mathrm{R}}=150 \mathrm{~s}$. (d) Pd nanosheets collected at $100{ }^{\circ} \mathrm{C}$ and $t_{\mathrm{R}}=150 \mathrm{~s}$. (e) $\mathrm{PdAu}$ nanostars obtained at $25^{\circ} \mathrm{C}$ and $t_{\mathrm{R}}=150 \mathrm{~s}$.

surface plasmon resonance (SPR) absorption at $740 \mathrm{~nm}$, (Fig. S8d $\dagger$ ). The SPR absorption of this nanostructure arises from the ultrathin nature because palladium nanorods obtained in air-liquid segmented flow did not exhibit any optical properties, contrarily to other noble metals ${ }^{4}$ (Fig. S8d $\dagger$ ). Consistent with effect of reactive gases, simple spherical Pd NPs resulted when $\mathrm{N}_{2}$ was used instead of $\mathrm{O}_{2}$ or $\mathrm{CO}$ in the slug flow experiments (Fig. S8a and $\mathrm{b} \dagger$ ).

Interfacial interactions that originate from electron transfer across the nanometer contact at the interface of two different materials can induce new properties that are not present in the individual components. In addition to the hetero nanostructures reported in this work by a single step synthesis, new nanostructures were obtained by a secondary growth method merging the same microfluidic reactor technology and ultrasound. ${ }^{51}$ Applying ultrasound, palladium nanorods were modified to obtain a dumbbell-like structure under a time scale process of $150 \mathrm{~s}$ and room temperature. The ultrasound induced cavitation promotes the synthesis of new nanostructured materials. ${ }^{52}$ Extreme physical and chemical conditions are created at localized spots while maintaining the bulk temperature. This property was crucial to conserve the metastable structure of nanorods and nanosheets, considering that these morphologies will be structurally modified at temperatures exceeding $80^{\circ} \mathrm{C}$. 
The MR was immersed in an ultrasonic bath operating at $42 \mathrm{kHz}$ and two streams of Pd nanorods and $\mathrm{Au}$ or Pd precursors, respectively, were injected. Au and Pd grew on the tips of the palladium nanorods by a heterogeneous nucleation-epitaxial growth process (Fig. $6 \mathrm{~b}$ and c). The selective growth at the ends of the Pd nanorods was accomplished by the growth of $\mathrm{Pd}$ and $\mathrm{Au}$ at the areas not covered by the capping agent anchored during the rod growth, facilitating the dumbbell like morphology. Au grew faster that Pd because the difference of reduction potentials between Pd and $\mathrm{Au}$ additionally promotes a galvanic replacement reaction. ${ }^{8,53}$ Regarding the etching effect of the galvanic replacement reaction, the palladium rod structure was still preserved and gold was simultaneously deposited at the ends, (Fig. 6c). This type of complex nanostructure could not be obtained using the galvanic replacement method at high temperature and in batch type reactor because the Pd rod would be split in several units and Oswald ripening would promote a semi-dumbbell structure. ${ }^{53}$

The combination of microfluidic and ultrasound resulted in growth of gold nanoparticles at the edges of the palladium nanosheets, producing a star-like nanostructure with potentially interesting optical and catalytic properties (Fig. 6e).

Carbon monoxide-liquid segmented flow also controlled the growth kinetics of platinum to produce cube-shaped nanostructures The critical variables for the formation of faceted platinum particles in short synthesis time were metal precursor and temperature. ${ }^{45}$ Mono-disperse Pt nanocubes with an average edge length of $5.6 \mathrm{~nm}$ and sharp corners were obtained using platinum(II) acetylacetonate at $200{ }^{\circ} \mathrm{C}$ and a residence time as small as 150 seconds (Fig. 7a). While faceted nanocrystals were obtained with $\mathrm{CO}$ segmented flow, only shapeless nanoparticles were obtained if $\mathrm{N}_{2}$ gas replaced CO (Fig. 7b). The binding strength and adsorption of $\mathrm{CO}$ on $\mathrm{Pt}$ facets were found to be crucial variables in formation of $\mathrm{Pt}$ nanocrystals with (100) facets. It worth noting that the synthesis of Pt cube shaped nanocrystals batch requires synthesis times ranging from 60 minutes ${ }^{54}$ to 20 hours ${ }^{55}$ demonstrating that microfluidic technology enable fast production of faceted nanocrystals.

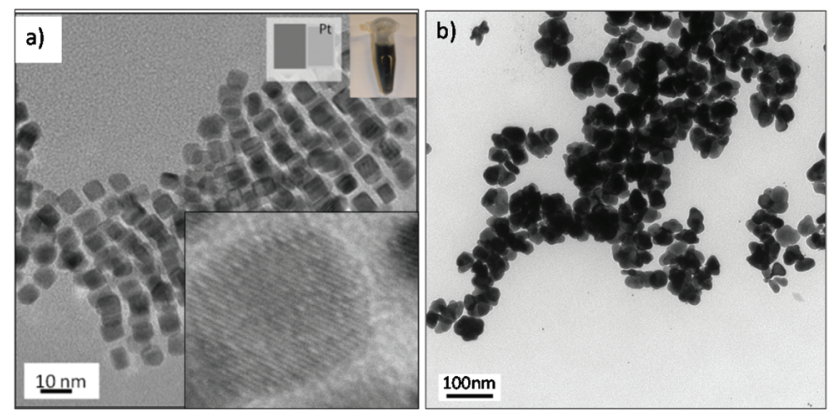

Fig. 7 TEM images of metallic platinum nanostructures obtained with platinum(॥) acetylacetonate at $200{ }^{\circ} \mathrm{C}, P_{\mathrm{co}}=120 \mathrm{psi}$ and residence time $=150$ s. under: (a) CO segmented flow. (b) $\mathrm{N}_{2}$ segmented flow.

\section{Experimental}

\section{Reagents}

Potassium bromide (KBr, Aldrich); dipotassium tetrachloroplatinate(II) $\left(\mathrm{K}_{2} \mathrm{PdCl}_{4}\right.$, Aldrich); platinum(II) acetylacetonate (Pt (acac) $)_{2}$ Aldrich); sodium tetracholoropalladate $\left(\mathrm{Na}_{2} \mathrm{PdCl}_{4}\right.$, Aldrich); silver nitrate $\left(\mathrm{AgNO}_{3}\right.$, Aldrich); nickel chloride(II) $\left(\mathrm{NiCl}_{2} \cdot 6 \mathrm{H}_{2} \mathrm{O}\right.$, Aldrich); cobalt chloride(II) $\left(\mathrm{CoCl}_{2} \cdot 6 \mathrm{H}_{2} \mathrm{O}\right.$, Aldrich); ruthenium chloride(III) $\left(\mathrm{RuCl}_{3} \cdot 3 \mathrm{H}_{2} \mathrm{O}\right.$, Aldrich), ascorbic acid (AA, Aldrich); sodium borohydride $\left(\mathrm{NaBH}_{4}\right.$, Aldrich); polyvinylpyrrolidone (PVP, MW = 55 000, Aldrich); tetradecyltrimethylammonium bromide (TTAB, Aldrich); ethylene glycol (EG, Aldrich); $N, N$-dimethylformamide (DMF, Omnisolv EMD); oleic acid (OLA, Sigma Aldrich), oleylamine (OAm, Across Organics). All chemical were of analytical grade and were used without further purification.

\section{Synthesis setup}

The reactants were introduced in the microfluidic reactor by three separated inlets, oxidant, reductant and a spare inlet that was blocked for case not requiring additional reagents. The reagents were introduced in the mixing zone by means of two high pressure syringe pumps (ISCO 100 DM), insuring good control of the flow rates and residence time. Before a reaction between the reagents, intimate contact between the component molecules must be realized through mixing either in laminar or segmented flow approach. The mixing zone was maintained at room temperature by circulation of cold water. The product stream exited the microreactor through the cold zone, quenching the nanoparticles growth. A back-pressure regulator (Jasco, Model BP-1580) maintained a set pressure inside the reactor while enabling samples to be collected in a continuous mode without depressurizing the system.

The microfluidic channels and ports were etched into silicon wafers by deep reactive ion etching (DRIE). The silicon surface was then oxidized to silica (glass) and anodically bonded to a Pyrex wafer to cap the channels, providing a glass surface that is compatible with reagents used. The reactor was designed to get a reaction volume of 100 microL. The nanostructures were analyzed by transmission electron microscopy (TEM, JEOL Model 200CX, HRTEM JEOL 2010 and STEM JEOl 2011F). The UV-Vis spectra was measured using an Agilent 8453 UV-Visible spectrophotometer.

Further details about the synthesis procedure of each type of nanostructure are given in the ESI. $\dagger$

\section{Conclusions}

In conclusion, we have demonstrated facile, fast and shapeselective synthesis of an extensive library of nanostructures in continuous flow process by using a microfluidic reactor platform. The microreactor separated the mixing and reaction in order to control the nucleation and growth kinetics, and it produced monodisperse NPs by controlling the residence time distribution. We avoided wall-induced nucleation and clogging 
by selection of flow pattern, reagents concentration, solvent, ligand, reducing agent and reaction conditions (temperature and residence time). Similar nanostructures to those reported for conventional batch-type reactor were realized at considerably reduced synthesis time along with new complex nanostructures: Pt-Ru, Pt-Ni and Pt-Co nanodendrites, Pt-Pd heterostructures, Ag-Pd core-shell NPs, Au-Pd nanodumbbells and $\mathrm{Au}-\mathrm{Pd}$ nanosheets. The fast synthesis with a fine control of size, shape and metal distributions was made possible by proper selection of metal precursors, reducing agents, stabilizing-capping agents, flow patterns, synthesis temperature and reactants residence time.

\section{Acknowledgements}

Authors acknowledge US NSF grant (CHE-0714189) for funding this research, and the MRSEC Shared Experimental Facilities at MIT, supported by the National Science Foundation under award number DMR-1419807 V. S. acknowledges the support of the Fulbright Commission and the Ministry of Education in Spain (Programa Nacional de Movilidad de Recursos Humanos. del Plan Nacional de I + D + I 2008-2011), as well as People Program (CIG-Marie Curie Actions, REA grant agreement no. 321642) to develop this research.

\section{Notes and references}

1 S. Kumar and T. Nann, Small, 2006, 2, 316-329.

2 G. J. Zhou, M. K. Lu, Z. L. Xiu, S. F. Wang, H. P. Zhang, Y. Y. Zhou and S. M. Wang, J. Phys. Chem. B, 2006, 110, 6543-6548.

3 V. Sebastian, M. Arruebo and J. Santamaria, Small, 2014, 10, 835-853.

4 Y. Xia, Y. J. Xiong, B. Lim and S. E. Skrabalak, Angew. Chem., Int. Ed., 2009, 48, 60-103.

5 C. B. Murray, D. J. Norris and M. G. Bawendi, J. Am. Chem. Soc., 1993, 115, 8706-8715.

6 S. Marre and K. F. Jensen, Chem. Soc. Rev., 2010, 39, 11831202.

7 P. M. Valencia, O. C. Farokhzad, R. Karnik and R. Langer, Nat. Nanotechnol., 2012, 7, 623-629.

8 L. Gomez, V. Sebastian, S. Irusta, A. Ibarra, M. Arruebo and J. Santamaria, Lab Chip, 2014, 14, 325-332.

9 R. L. Hartman, J. R. Naber, N. Zaborenko, S. L. Buchwald and K. F. Jensen, Org. Process Res. Dev., 2010, 14, 13471357.

10 J. Baek, P. M. Allen, M. G. Bawendi and K. F. Jensen, Angew Chem., Int. Ed., 2011, 50, 627-630.

11 S. Marre, J. Park, J. Rempel, J. Guan, M. G. Bawendi and K. F. Jensen, Adv. Mater., 2008, 20, 4830-4834.

12 A. J. Brown, N. A. Brunelli, K. Eum, F. Rashidi, J. R. Johnson, W. J. Koros, C. W. Jones and S. Nair, Science, 2014, 345, 72-75.
13 F. Cacho-Bailo, G. Caro, M. Etxeberria-Benavides, O. Karvan, C. Tellez and J. Coronas, Chem. Commun., 2015, 51, 11283-11285.

14 Y. H. Kim, L. Zhang, T. Yu, M. S. Jin, D. Qin and Y. N. Xia, Small, 2013, 9, 3462-3467.

15 A. Larrea, V. Sebastian, A. Ibarra, M. Arruebo and J. Santamaria, Chem. Mater., 2015, 27, 4254-4260.

16 S. E. Lohse, J. R. Eller, S. T. Sivapalan, M. R. Plews and C. J. Murphy, ACS Nano, 2013, 7, 4135-4150.

17 L. Zhang and Y. N. Xia, Adv. Mater., 2014, 26, 26002606.

18 L. Zhang, G. D. Niu, N. Lu, J. G. Wang, L. M. Tong, L. D. Wang, M. J. Kim and Y. N. Xia, Nano Lett., 2014, 14, 6626-6631.

19 V. Sebastian, S. Basak and K. F. Jensen, AIChE J., 2016, 62, 373-380.

20 M. Thiele, J. Z. E. Soh, A. Knauer, D. Malsch, O. Stranik, R. Muller, A. Csaki, T. Henkel, J. M. Kohler and W. Fritzsche, Chem. Eng. J., 2016, 288, 432-440.

21 A. Knauer, A. Csaki, F. Moller, C. Huhn, W. Fritzsche and J. M. Kohler, J. Phys. Chem. C, 2012, 116, 9251-9258.

22 S. Marre, A. Adamo, S. Basak, C. Aymonier and K. F. Jensen, Ind. Eng. Chem. Res., 2010, 49, 11310-11320.

23 V. S. Cabeza, S. Kuhn, A. A. Kulkarni and K. F. Jensen, Langmuir, 2012, 28, 7007-7013.

24 S. K. Lee, X. Y. Liu, V. S. Cabeza and K. F. Jensen, Lab Chip, 2012, 12, 4080-4084.

25 S. A. Khan, A. Gunther, M. A. Schmidt and K. F. Jensen, Langmuir, 2004, 20, 8604-8611.

26 P. D. Yang, H. Lee, S. E. Habas, S. Kweskin, D. Butcher and G. A. Somorjai, Angew. Chem., Int. Ed., 2006, 45, 78247828.

27 H. I. Schlesinger, H. C. Brown, D. L. Mayfield and J. R. Gilbreath, J. Am. Chem. Soc., 1953, 75, 213-215.

28 J. Polte, R. Erler, A. F. Thunemann, S. Sokolov, T. T. Ahner, K. Rademann, F. Emmerling and R. Kraehnert, ACS Nano, 2010, 4, 1076-1082.

29 A. Gunther, M. Jhunjhunwala, M. Thalmann, M. A. Schmidt and K. F. Jensen, Langmuir, 2005, 21, 15471555.

30 J. M. Kohler, S. N. Li and A. Knauer, Chem. Eng. Technol., 2013, 36, 887-899.

31 L. Uson, V. Sebastian, A. Mayoral, J. L. Hueso, A. Eguizabal, M. Arruebo and J. Santamaria, Nanoscale, 2015, 7, 1015210161.

32 M. L. Wu, D. H. Chen and T. C. Huang, Langmuir, 2001, 17, 3877-3883.

33 W. B. Hou, N. A. Dehm and R. W. J. Scott, J. Catal., 2008, 253, 22-27.

34 C. R. Henry, Surf. Sci. Rep., 1998, 31, 235-325.

35 R. Adzic, M. H. Shao, P. Liu and J. L. Zhang, J. Phys. Chem. $B, 2007,111,6772-6775$.

36 R. D. Tilley, S. S. Cheong and J. D. Watt, Nanoscale, 2010, 2, 2045-2053.

37 Y. J. Xiong, J. Y. Chen, B. Wiley, Y. N. Xia, Y. D. Yin and Z. Y. Li, Nano Lett., 2005, 5, 1237-1242. 
38 M. Luo, H. W. Huang, S. I. Choi, C. Zhang, R. R. da Silva, H. C. Peng, Z. Y. Li, J. Y. Liu, Z. K. He and Y. N. Xia, ACS Nano, 2015, 9, 10523-10532.

39 L. Wang and Y. Yamauchi, J. Am. Chem. Soc., 2009, 131, 9152-9153.

40 L. Wang and Y. Yamauchi, Chem. Mater., 2009, 21, 3562-3569.

41 H. Lee, S. E. Habas, S. Kweskin, D. Butcher, G. A. Somorjai and P. D. Yang, Angew. Chem., Int. Ed., 2006, 45, 7824-7828.

42 A. Mohanty, N. Garg and R. C. Jin, Angew. Chem., Int. Ed., 2010, 49, 4962-4966.

43 B. Lim, M. J. Jiang, P. H. C. Camargo, E. C. Cho, J. Tao, X. M. Lu, Y. M. Zhu and Y. A. Xia, Science, 2009, 324, 13021305.

44 B. Lim, M. J. Jiang, T. Yu, P. H. C. Camargo and Y. N. Xia, Nano Res., 2010, 3, 69-80.

45 V. Sebastian, C. D. Smith and K. F. Jensen, Nanoscale, 2016, 8, 7534-7543.

46 B. Lim, M. J. Jiang, J. Tao, P. H. C. Camargo, Y. M. Zhu and Y. N. Xia, Adv. Funct. Mater., 2009, 19, 189-200.
47 Y. J. Xiong, H. G. Cai, B. J. Wiley, J. G. Wang, M. J. Kim and Y. N. Xia, J. Am. Chem. Soc., 2007, 129, 3665-3675.

48 X. Q. Huang, S. H. Tang, X. L. Mu, Y. Dai, G. X. Chen, Z. Y. Zhou, F. X. Ruan, Z. L. Yang and N. F. Zheng, Nat. Nanotechnol., 2011, 6, 28-32.

49 P. F. Siril, L. Ramos, P. Beaunier, P. Archirel, A. Etcheberry and H. Remita, Chem. Mater., 2009, 21, 5170-5175.

50 J. B. Wu, A. Gross and H. Yang, Nano Lett., 2011, 11, 798802.

51 D. G. Shchukin, D. Radziuk and H. Mohwald, Annu. Rev. Mater. Res., 2010, 40, 345-362.

52 J. H. Bang and K. S. Suslick, Adv. Mater., 2010, 22, 10391059.

53 P. H. C. Camargo, Y. Xiong, L. Ji, J. M. Zuo and Y. Xia, J. Am. Chem. Soc., 2007, 129, 15452-15453.

54 B. H. Wu, N. F. Zheng and G. Fu, Chem. Commun., 2011, 47, 1039-1041.

55 J. T. Ren and R. D. Tilley, J. Am. Chem. Soc., 2007, 129, 3287-3291. 